\title{
Superluminal Motion is Possible: Discussion of Limitation and Approximation of Special Relativity
}

\author{
Zihua Zhang \\ Box 171 Bupt Beijing 100876 China \\ Apt.22-441, No.10 Xi Tu Cheng Lu, Haidian District, \\ Beijing 100876, China \\ Zhangzihua01@126.com
}

\author{
Zhiying Zhong \\ Box 171 Bupt Beijing 100876 China \\ Apt.22-441, No.10 Xi Tu Cheng Lu, Haidian District, \\ Beijing 100876, China
}

\begin{abstract}
Here discussed the difference between Special Relativity and Newton Mechanics from the epistemology; pointed out they discuss real and visual law of body motion respectively, and the Special Relativity is only valid approximately for $v<<c$; also proved The conclusion: no body can move faster than $c$ is wrong and "rule contraction" and "Clock retardation" are rainbow. And pointed out that fly velocity of particle (neutrino) larger than $c$ and superluminal communications are possible.
\end{abstract}

Keywords-superluminal motion; Special Relativity; epistemology; Visual error

\section{INTRODUCTION}

Recently superluminal phenomena appear often [1], such as: the departure velocity of celestial bodies is lager than $5 \mathrm{c}$ observed in Radio Astronomy [2]; Transmission speed of light pulse can larger than c, even has a negative value and time advance occurs [3,4]; The flying speed of particle (neutrino) can faster than c [5] also be observed by OPERA Scientists. All of these phenomena are disputed, since they contradict with the conclusion of Einstein: no body (including information) can move faster than $c$ [6] or Somerfield and Brillouin's: the velocity of energy transport must less than c [7]. We deal with what reason caused such contradiction recently and introduce some results here. We think this contradiction comes from the misunderstanding of the special Relativity and the superluminal motion of body is a natural phenomenon [8], since that is promised by objective epistemology. Any physical theorem and law only validate for a certain range and condition [9].

\section{THEORETICAL BASES FOR SUPERLUMINAL PHENOMENA.}

Since 1905 Einstein published the Special Relativity (SR) [10], especially after three predictions of the General Relativity were demonstrated by astronomic observation [11]. The Relativity was considered as absolute true, also can guiding the classical Mechanics and all physical phenomena research by some persons. Then Brillouin deduced ve/c must less then c, where ve is the velocity of energy transport. However the superluminal phenomena observed often violate the above theory. The reason caused such violation have to search for from the epistemology.

\section{A. Two Kinds of Epistemology with Difference Applied Range [11].}

As we know, in practice of human being, about how to cognize the body in external world, there are two kinds of viewpoint or methods i.e. epistemology or theory of knowledge generally: They are Objective and Subjective (or visual) theory of knowledge.

1) Objective theory of knowledge. According to such theory of knowledge, in external (real) world the body studied exists objectively and independent of our observation; the cognizing body also not spend time. Most scientific theories including Newton Mechanics and Quantum Mechanics belong to such theory of knowledge. In this theory of knowledge, time-space quantities of the body are independent, the relationship between them in different initial systems is determined by Galilean transformation, we have

$$
x^{\prime}=x \pm v t, y^{\prime}=y, z^{\prime}=z, t^{\prime}=t
$$

sign "+"and"-" are determined by the direction of velocity $\mathrm{v}$ between two systems; the Clock is synchronous in all systems, the simultaneity is absolute. The character of such theory is that we deal with the body itself always; the exact position of body is independent of the choice of reference frame.

2) Subjective (visual) theory of knowledge. This theory tells us we affirm the existence of the body in external world due to receiving a signal sent from it and producing a image in eye. The signal is transferred by light or electromagnetic wave and form the body to observer spends a certain time $\square \mathrm{t}=\mathrm{L} / \mathrm{c}$, where $\mathrm{L}$ is the distance between the body and the observer; $c$ is the light speed. The SR just belongs to such theory of knowledge. This kind of theory is based on directly detect body by virtue of light. For convenience of discussion, suppose the light speed is constant and equals to $\mathrm{c}$, since in fact, the light speed varies and depends on the electro-magnetic character of media and the state of observer, so that the discussion of the Special 
3) Relativity only limited in vacuo. In such theory, the time-space quantities of the body are connected by the light speed c, they constitute a four dimension space, the simultaneity is relative and judged by the observer, and the body studied is that recognized by our vision. The relationship between time-space quantities in different initial systems is determined by Lorentz transformation, and have

$$
x^{\prime}=\frac{x \pm v t}{\sqrt{1-(v / c)^{2}}}, \quad t^{\prime}=\frac{t \pm \frac{v}{c^{2}} x}{\sqrt{1-(v / c)^{2}}}, \quad y^{\prime}=y, \quad z^{\prime}=z
$$

sign " +" or " -" is determined by the velocity direction too. Since the value of the light speed $\mathrm{c}$ is limited, the time needed for transferring the signal of body to observer is $\square \mathrm{t}=\mathrm{L} / \mathrm{c}$. The character of the subjective epistemology is only for still body the object observed is body itself, for a moving body the object observed is an image of body or a virtual body which constitutes a viewed (or virtual) world, since the body has left from original position (Fig. 1). The difference of position between real and virtual body is $\square \mathrm{L}=\mathrm{L} \square \mathrm{v} / \mathrm{c}$, where $\mathrm{v}$ is the velocity of the body and a vector. The difference of the body form (including time and position) between the real and observed body is called as the visual error which is just as the position of a fish in water (Fig.2). This difference of the body form due to the body motion and the light speed is limited also be further amplified by Lorentz transformation, so that the difference of body form depends not only on the velocity of body motion and the distance $\mathrm{L}$ but also on the choice of coordinate system. Therefore the Special Relativity is an "imaginary" or "phenomenal" theory and belongs to the Subjective or visual epistemology, since you only cognize the body which be seen. The astronomic observation just is a representative of such theory of knowledge. Obviously the world seen is different from the real world and is virtual. So that the subjective theory of knowledge can't study the real motion of body with larger velocity, only study the visual law of body motion. We think the Special Relativity is a visual Mechanics [11] really which deals with the law of the image motion.

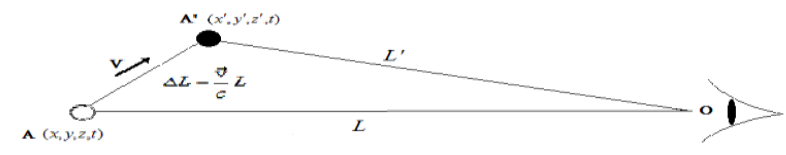

Figure 1. Visual error of position $\Delta \mathrm{L}$ due to value of light speed $\mathrm{c}$ is limited. A'--real position of body; A---- visual position of body.

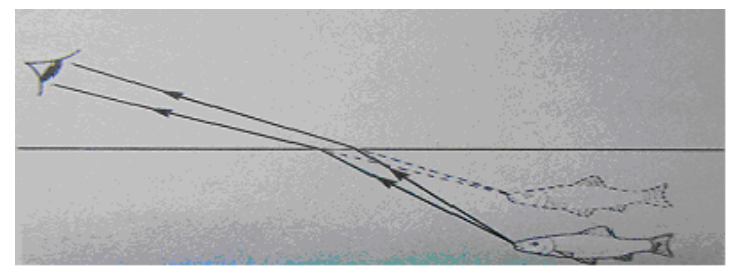

Figure 2. Visual and real position of a fish in water.

\section{B. The Problem of Reality of Four-Dimension Space in Special Relativity.}

Some persons think the real law of body motion, especially for faster body should be studied in fourdimension space that represents the real external world. But we don't think so. The reason is that in fact this fourdimension space i.e. the viewed world is virtual and constructed by images and the principle of invariance of the light velocity is invalid in media due to the dragging effect of velocity, which also exists in air [23] has been proved, and only approximately valid in vacuo. Exactly speaking the Einstein's paradox [10], If you go forward with a light line A and look at a companying light line $\mathrm{B}$, the electric field $\mathrm{E}$ in line B will only vibrates at same place, is correct, that has been proved by the experiment of interference of light, since if this paradox is incorrect as Einstein thought the light line B still propagate with speed $c$, the phase difference between two beams will change rapidly, by means of combining light line $\mathrm{A}$ and $\mathrm{B}$ will no interference fringe appear, obviously it is wrong; but if you go forward with line A with a velocity $\mathrm{v}<<\mathrm{c}$, the light line $\mathrm{B}$ will propagate with speed $\mathrm{c}$ in any direction is correct approximately, because of the value of the light velocity is independent of light souse, but depends on the state of observer that has been neglected by Einstein. So that the principle of invariance of light velocity, then the Lorentz transformation validates only for $\mathrm{v}<<\mathrm{c}$ even if in vacuo, and in media must have $v=0$, it evolves to Galilean transformation. For evidence, the value of light speed measured in four-dimension space is infinite; when a body moves faster than $\mathrm{c}$, the measured value of velocity is negative deviating from its real value seriously; but when the velocity much less than $\mathrm{c}$ the measured value in fourdimensional space is near its exact value too. The value $\mathrm{c}=2.99792458 \square 108 \mathrm{~m} / \mathrm{s}$ and the physical constant used in Relativity are measured in Galilean transformation system. But in the explaining Astronomic and other observed phenomena, the Relativity is better than Classical Mechanics due to the considering the "visual error" and the most velocity of celestial body is much less than c, for example the velocity of solar system moving around the Milky Way galaxy is about $2.3 \square 105 \mathrm{~m} / \mathrm{s}$. The celestial bodies seen in sky are only their images or virtual due to value of $\mathrm{c}$ is limited. The $\square \mathrm{t}$ and $\mathrm{L}$ vary in very lager range, such as for the Moon, $\square \mathrm{t} \sim 1.5 \mathrm{~s}$ and for the Sun $\square \mathrm{t} \sim 2.5$ minute, for another celestial bodies $\Delta \mathrm{t}$ may be tens thousands to several million years, the position difference $\square \mathrm{L}=\mathrm{v} \square \mathrm{t}$ for small $\Delta \mathrm{t}$ or $\Delta L=\int_{0}^{\Delta t} \vec{v} d t$ when $\square \mathrm{t}$ is lager, where $\vec{v}$ is the velocity of celestial body. So that the difference of the body form leads the structure diagram of celestial bodies in Astronomy is very different from that in space navigation that belongs to objective epistemology. The other important result in SR is so-called "rule contraction" and "Clock retardation". Most persons think these phenomena really happen, but in our opinion they have only apparent meaning or are rainbow. If we have two systems $\mathrm{K}$ and $\mathrm{K}$ ' with relative velocity $\mathrm{v}$ and time-space quantities $(x, t),\left(x^{\prime}, t^{\prime}\right)$, according to Lorentz transformation and its adverse transformation we have [12] 


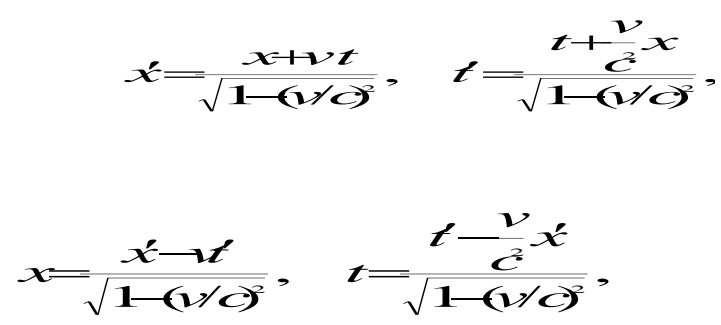

In system $\mathrm{K}$ we have

$$
x_{2}-x_{1}=\left(x_{2}^{\prime}-x_{1}^{\prime}\right) \sqrt{1-(v / c)^{2}}, t_{2}-t_{1}=\left(t_{2}^{\prime}-t_{1}^{\prime}\right) / \sqrt{1-(v / c)^{2}} .
$$

And in system $\mathrm{K}^{\prime}$ we still have

$$
x_{2}^{\prime}-x_{1}^{\prime}=\left(x_{2}-x_{1}\right) \sqrt{1-(v / c)^{2}}, t_{2}^{\prime}-t_{1}=\left(t_{2}-t_{1}\right) / \sqrt{1-(v / c)^{2}} .
$$

In fact, the principle of Relativity tells us the rule or Clock is equivalent in each initial system. So that we think the socalled time-space be curved is just an apparent phenomena, just like as river gage the part in water is shorted or when you sit in a faster moving train and look outside, the earth twirls likely. In fact it is a display of visual error. We have to know that the viewed world is different from the real world in some situation.

\section{The Conclusion of Einstein's Limited Velocity is Wrong}

As we know, Einstein got the conclusion of limited velocity by means of visual epistemology was based on three reasons: 1), If $v>c$, the violation of causality will happen; 2 ), If $\mathrm{v}=\mathrm{c}$, the energy will be infinite; 3 ), According to theorem of velocity addition, $v$ can't larger than $\mathrm{c}$. We can prove these reasons are invalid also for the real world.

1) during $\mathrm{v}>\mathrm{c}$, the denominator in Lorentz transformation is imaginary, $x^{\prime}, t^{\prime}$ are imaginary number, but $x$ and $t$ are real number, we have

When $\mathrm{x}$ is constant,

$$
t_{2}^{\prime}-t_{1}^{\prime}=\frac{t_{2}-t_{1}}{i \sqrt{(v / c)^{2}-1}} \text {. }
$$

$$
x_{2}^{\prime}-x_{1}^{\prime}=\frac{x_{2}-x_{1}}{i \sqrt{(v / c)^{2}-1}} \text {. }
$$

When $\mathrm{t}$ is constant,

The violation of causality not happen, But in the experiment of superluminal propagation of light pulse, when $\operatorname{vg}<0$, the time advance happen which is determined by the position of pulse peak is at $\mathrm{z}$-vgt=0[13], we have discussed this question in paper [9].

2) when $v=c$, is energy infinite? As we know the point $\mathrm{v}=\mathrm{c}$ is strange point for both visual epistemology and Lorentz transformation, in fact the real kinetic energy of body is determined in Galilean transformation, not in Lorentz transformation since the body form in fourdimension space is virtual. $\mathrm{v}$ can't equal to $\mathrm{c}$ is determined by the Lorentz transformation not by the real energy itself.
Strictly, the real motion law of body is studied in classical Mechanics then when $\mathrm{v}=\mathrm{c}$ no energy infinite problem.

3) Einstein called formula

$$
u=\frac{u^{\prime}+v}{1+u^{\prime} v / c^{2}}
$$

as the relativistic addition theorem of velocity [12]. Since $\mathrm{u}^{\prime}=\mathrm{dx} / \mathrm{dt}$ ' and $\mathrm{u}=\mathrm{dx} / \mathrm{dt}$ are value of same velocity in different system, the units using to measure time $t 、 t^{\prime}$ and distance $\mathrm{x} 、 \mathrm{x}$ ' are different due to the Lorentz transformation. To assert that $\mathrm{v}$ always less than $\mathrm{c}$, Einstein adopted the idea of objective theory of knowledge i.e. Galilean transformation's principle, and also assumed the unit of velocity are same, that means all velocities is just in same system. We have to know that the time space quantities in different systems in Special Relativity only have a corresponding relationship, they are object and image for each other, and can't put them together via plus or minus or directly compare. Furthermore u' and $u$ are just value of one velocity in different coordinate systems. In our opinion the formula (8) should be called as the correspondence principle of velocity. The plus or minus of velocities only can be done in same system with same measuring unit. It belongs to Galilean transformation category. The meaning of equ. (8) is that: In vacuo, if the velocity of body is u' in one system; and in other system with relative velocity $\mathrm{v}$ to that the observed value of velocity is $u$. The relation between $u$ ' and $u$ is object and image.

Even if the conclusion of limited velocity is suitable for visual epistemology, moreover extending that to objective epistemology is incorrect since two epistemologies coexist, and have different applied range (real or virtual world corresponding to cause and effect).

\section{EXPERIMENTAL VERIFICATION OF SUPERLUMINAL PHENOMENA}

The pioneer experiment for verifying the superluminal propagation of light was made by Wood in 1904 [14] when observing the anomalous dispersion in Sodium vapor. He discovered the yellow light has the refractive index $\mathrm{n}<1$, means that the propagation velocity vp larger than $\mathrm{c}$, and this result was interpreted by harmonic oscillator model of refractive index [7] successfully. Because of Einstein's conclusion no body can move faster than c, Wood's experimental result was not got enough attention. Until to the eighties of last century, Astrophysicists observed the departure velocity of two sub-sources of radio-sources 3C273 outside of the Milky Way galaxy is larger than 5c [2]. This phenomenon contradicts with the SR and catches Scientist's attention. Recently beyond the astrophysics such as in electro-magnetic radiation (include light) and basic particle area also observed the superluminal phenomena. Here list some examples. In 1992 G. Nimtz, Prof. Of Colonge University of German, et al discovered that the microwave pulse passes through a barrier with a group velocity $\mathrm{vg}=4.7 \mathrm{c}$ by means of double microwave pulses race [15]. In 1993 American Chinese Scientist L. Y. Chiao et al 
[16] observed the velocity of photon penetrating a barrier is $1.7 \mathrm{c}$ by means of double photons race. In $2000 \mathrm{~L}$. J. Wang et al [3] demonstrated the light pulses propagate in a transparent anomalous medium $(6 \mathrm{~cm} \mathrm{Cs}$ cell) the group velocity $v g=-c / 310$, and a time advance $-63 \pm 1 \mathrm{~ns}$ happen. Chinese scientists also observed superluminal phenomena, $\mathrm{Z}$. $X$ Huang et al [17] in the double high frequency pulses race experiment observed vg about $1.95 \mathrm{c}$ in 2003; X. Z. Chen et al [18] observed when the light pulse passes through a $6 \mathrm{~cm}$ Cs cell time advance equals to 0.9 s corresponding to group velocity vg $=-c / 4500$ in 2004, and L. Zhang [19] et al also demonstrated the light pulses propagate in $10 \mathrm{~m}$ Optical Fiber based on Brillouin Oscillation with group velocity $\mathrm{vg}=$ $0.15 \mathrm{c}$ and time advance $\mathrm{t}=-221.2 \mathrm{~ns}$. Especially in 2011 OPERA Scientists $[2,20]$ found the flying speed of Neutrino is faster than c, through $730 \mathrm{~km}$ the spend time less than light is 60ns, and this result had been re-verified, up to now many scientists still doubt this result, but in fact in 1987 during observing the explosion of supernova SN1987A had found that the time arriving earth of Neutrino is shorter than light about 4 hours, means that the flying speed of Neutrino is faster than c exactly. According to OPERA scientists said the result, neutrino faster than $\mathrm{c}$ be observed more than ten thousand times, it is enough to say particle neutrino can move faster than c certainly.

\section{INFLUENCE OF SUPERLUMINAL STUDY ON THE DEVELOPMENT OF SCIENCE AND TECHNOLOGY}

Actually the objective and subjective theory of knowledge coexist in the world, and they have different applied range and are mutual complement and contact, the connection point is at $\mathrm{v}=0$, i.e. for still body. The objective theory of knowledge studies the real law of body motion due to always deal with the body itself and the Subjective theory of knowledge only studies the visual law of body motion since only deal with the image of body. The difference between them is called as visual error, and the faster velocity of body motion, the larger difference between them. So that we think The Relativity can't guide the Classical Mechanics, since they belong to different category. The Astronomic observation belongs to the subjective theory of knowledge so that the celestial structure diagram obtained by Astronomic observation is very different from that needed by the space navigation due to they belong to different category of epistemology. Classical and Quantum Mechanics belong to category of the objective theory of knowledge accepting the concept of superluminal motion but can't interpret some observing phenomena. Einstein developed a new theory - the Relativity by means of the subjective theory of knowledge and explained some observing phenomena triumphantly and also obtained the relationship between mass and energy, especially after three predictions of General Relativity was demonstrated [10], the Relativity was considered as an absolute truth, due to lack the understanding the essence of theories and applied range, led the progress of Physics from one extreme to another, made the serious repression on that only can be interpreted by the objective theory of knowledge. The conclusion of limited velocity of Einstein restricts that human being cognize the external world rightly, leading a series of mistake in physics that we discussed in other paper $[12,13]$. Here only list some examples.

1) Under the influence of the SR, the theory of wave propagation and group velocity proposed by Sommerfend and Brillouin [7] complicated the transmission theory of light and information. For example they thought the concept of group velocity not validate in the region with larger anomalous dispersion, in this region the group velocity no longer represents the velocity of a signal or energy transport, and suggested the concepts of five velocities. WKD experiment of superluminal propagation of light pulse [2] was denied and Nimtz's superluminal propagation of information [15] was denied too. As we know, the concept of photon trap has been accepted but velocity can faster than c was refused; apparently the light propagation velocity only can slow down and can't fast up [21]. The superluminal light pulse was considered as visual error contrarily. In fact the superluminal light pulse can be detected means these pulses are real and exist objectively. We have pointed out ${ }^{[9]}$ that the inner energy of molecules or atoms not take part in the energy transport, and shown the curve $\mathrm{v}_{\mathrm{e}} / \mathrm{c}$ in Brillouin's figure is a curve of efficiency of energy transport, and corresponds to the contour of spectral line. The front velocity can't realized, and the signal velocity equals to the phase velocity of the load wave in analog communications, and equals to group velocity in digital communications. Both of them can larger than c.

2) We have proved [12] that the result of WKD experiment is correct in theory, the group velocity is just the velocity of pulse peak with argument $\mathrm{z}-\mathrm{v}_{\mathrm{g}} \mathrm{t}=0$; the difference between phase and group velocity is due to the dispersion, and have

$$
v_{g}=v_{p}-\lambda \frac{d v_{p}}{d \lambda}, n_{g}=n+\omega \frac{d n}{d \omega}
$$

If dispersion $\mathrm{D}=\mathrm{dn} / \mathrm{d} \omega=0$, we have $\mathrm{v}_{\mathrm{g}}=\mathrm{v}_{\mathrm{p}} ; \mathrm{D}>0, \mathrm{n}_{\mathrm{g}}>\mathrm{n}$, and $v_{\mathrm{g}}<\mathrm{v}_{\mathrm{p}}$, when $\mathrm{v}_{\mathrm{g}}$ very small the photon trap happen; $\mathrm{D}<$ 0 , we have $n_{\mathrm{g}}<\mathrm{n}$, and $\mathrm{v}_{\mathrm{g}}>\mathrm{v}_{\mathrm{p}}$. When $\propto>\mathrm{v}_{\mathrm{g}}>\mathrm{c}$ it corresponds to ordinary superluminal. When $\mathrm{n}_{\mathrm{g}}<0$, and $\mathrm{v}_{\mathrm{g}}<0$, the time advance happen, it corresponds to anomalous superluminal. For WKD experiment, cell length $\mathrm{z}=6 \mathrm{~cm} ; \mathrm{v}_{\mathrm{g}}=-\mathrm{c} / 310$, we calculated $\mathrm{t}=-62 \mathrm{~ns}$ [21], coincided with measured value $63 \pm 1 \mathrm{~ns}$ very well. We also calculated for input light pulse, the dispersion length $L_{D}=23 \mathrm{~m} \mathrm{[22],} \mathrm{so} \mathrm{that} \mathrm{passing} 6 \mathrm{~cm}$ cell the output pulse has no distortion.

3) we think that the principle of invariance of light velocity is the soul of the SR, and the Lorentz transformation is the core of the SR. Due to the dragging effect of velocity the Lorentz transformation is invalid in medium (include air [23]), even if in vacuo the Lorentz transformation is valid only for $\mathrm{v}<<\mathrm{c}$ since light velocity depends on the state of observer. If expanding the discussion of the SR to complex domain, when $v>c$, the time space quantities will be a complex number, put that in the expression of wave, just lead this wave has an attenuation or gain, such as radiation mode and stability of 
modulation in fiber optics [24] or Cerenkov radiation. But Einstein only limited the discussion of the SR in real domain so as to get an incorrect conclusion: v can't larger than c. We think that, the electro-magnetic character of the medium determines the propagation velocity of electro magnetic wave, and the velocity of the particle is determined by motion law itself and the condition of generation and has nothing to do with The SR.

4) at present, the signal transmission velocity is about c for mobile communications, and equals to $\mathrm{c} / \mathrm{n}_{1}$ for fiber communications, here $\mathrm{n}_{1}$ is refractive index of fiber core. These velocities almost are constant. We think that the communications just like as traffic; the capacity of communication system is ratio to the transmission speed of signal. If the superluminal light pulses are employed to transfer the information, the operation speed of computer will raise and a single channel is enough to get a high bit rate, the communication system will be simplified; when the group velocity of light pulses are negative, we can make a "time compensator" to short the time of information transmission [9]. We have proved that by means of the measure of double gain line and suitable choice the width and the space of gain peaks the superluminal propagation of information in fiber also can be done keeping away from the distortion of signal [23]. It is worthy to obtain a transparent medium with higher negative dispersion for the development of Informatics.

\section{SUMMARY}

Here we introduced recent results on superluminal phenomena briefly, pointed out that the principle of invariance of light velocity is valid for $\mathrm{v}<<\mathrm{c}$ in vacuo approximately. The Special Relativity is only study the visual law of body motion with $\mathrm{v}<<\mathrm{c}$. The real law of body motion is studied by means of Objective theory of knowledge. Superluminal motion is a natural phenomenon [8], superluminal communication is possible [25]. By means of the Special Relativity to deny the existence of superluminal phenomena is incorrect. Classical (Newton) Mechanics, Quantum Mechanics and Visual Mechanics (Relativity) are three kinds of theory studying the motion law of body, they have different applied range and object been studied. Classical Mechanics and Quantum Mechanics belong to objective theory of knowledge, and admit the superluminal phenomena. We can say that any physical theorem and law has its deduced condition and different applied range, before distinguishing what you are studying, and what category belongs to this study to judge what experimental result is correct or incorrect or what experiment phenomena can or can't happen is unscientific, that is our opinion and would like discuss our viewpoint with everyone. In our opinion, we have to distinguish what is the real world and visual world strictly, and recognize the limitation and approximation of the Relativity.

\section{REFERENCES}

[1] Huang. Z. X, Theory and Experiments of Faster than Light Research, (in Chinese) (cience express, Beijing, 2005)

[2] Cyclopedia of China, Vol. 1, (Cyclopedia Press, 1994, Peking); nature journal, No.1, 1980.

[3] L. J. Wang, A. Kuzmich \& A. Dogariu; Nature, 406, 277 (2000).

[4] Dogariu. A, Kuzmich. A, and Wang. L. J, Transparent anomalous dispersion and superluminal light-pluse propagation at a negative group velocity, Phys. Rev. A, Vol. 63,053806

[5] Reference News, 2011-9-27, 2011-9-27, 2011-9-29, p.7.2011-11-20, p.7. (Nature 2011-9-22)

[6] Einstein. A, The principle of Relativity and Its Conclusion, Einstein's Corpus, Vol.2 (Business Press, 1977 Peking). 151.

[7] Brillouin. L, Wave Propagation and Group Velocity, (Academic Press, New York and London, 1960).

[8] Cyclopedia of China, (Electronic engineering and Electrotechnics) (Cyclopedia Press, 2012, Jan. Peking), p.35.

[9] Zhang zi hua, Zhang hua an, Study of Superluminal Phenomena and Its influence on Physics and Informatics, 6th annual meeting of photonics of China. Oct. 2008, ZhongQingChinese Scientist, 2012, No.08,p.80-89.

[10] A. Einstein, Ann. d. Phys. 17. 891 (1905),A. Einstein, Relativity, (in Chinese, translated by Yi Hong Bo, Li Zhi Mou), Press of Jiangsu People, 2011, Jan.)

[11] Zhang zi hua, Zhong zhi ying and Zhang hua an, Simple explanation of contradiction between Special Relativity and Superluminal phenomena, Chinese Scientist, 2012 No.6.

[12] Ni. G. J. and Li. H. F. Modern Physics, (Shanghai Science and Technology Press, 1979)

[13] Zhang zi hua, Reshape and Superluminal propagation of light pulse in negative dispersion media, Chinese Scientist, 2011,No.1,p.1.

[14] Jenkins F. A, and White H. E, Fundamentals ofPhysical Optics (1937) (in Chinese Translated by Phy. Dept. of Qinghua University), Chp. 9.

[15] Nimtz G, Heitmann W. Superluminal photonic tunneling and quantum electronics. Prog Quam Electr, 1997, 21(2), 81 108.

[16] Steinberg A. M, Kwiat P. C, Chiao L. Y; Measurement of the single photon tunneling time. Phys. Rev. Lett, 1993, 71(5), 708-711.

[17] Huang Z. X, Lu G. Z, Guan J. Superluminal and Negative group velocity in electro-magnetic wave propagation. Engineering sciences, 2003, 1(2), 35-39.

[18] Chen X. Z, Xiao F, Li L. M et al; Acta of Broadcast college of Beijing (Nature Science), Supplement, 2004, 11; 19-26.

[19] Zhang L, Zhan L, et al, SuperluminalPropagation at Negative Group Velocity in Optical Fibers Based on Brillouin Lasing Oscillation, Phy. Rev. Lett. 107, 093903,(2011).

[20] Wikipedia, entry, Neutrino.

[21] Zhang. Z. H. and Zhang H. A, Superluminal phenomena and Superluminal Communications, Analects of reformation, development and innovation of China, (History and literature of Chinese party Press, Oct. 2008), p.533.

[22] Zhang zi hua, distortionless propagation of superluminal pulses in gain-added media, "Shen Shi Zhi Guang- Theory and Practice of Present innovation of China, " Oct. 2009

[23] Z. Y. Wang, P. Y. Wang, and Y. R. Xu, Crucial experiment to resolve Abraham-Minkowski controversy, Optik 2011, 122, 1994-1996

[24] G. P. Agrawal, Nonlinear Fiber Optics and Its Application (in Chinese translated by Jia D.F. et.al.) (Publishing House of Electronics Industry, 2010, Beijing,)

[25] Zi hua Zhang, Special way of Optical Communication, the technique of Superluminal Communications, - IWFCN 2011 conferences. Dec. 11, 2011. Hong Kong. Instrumentation Measurement circuit and systems I (ADVANCES IN INTELLEGIENT AND SOFT COMPUTING 127), (Springer 2011) .145-53, 